\title{
Archipel
}

ARCHIPEL Études interdisciplinaires sur le monde insulindien

$97 \mid 2019$

Varia

\section{Urip Iku Urub : Untaian Persembahan 70 Tahun Profesor Peter Carey}

\section{Henri Chambert-Loir}

\section{(2) OpenEdition \\ 12 Journals}

\section{Electronic version}

URL: https://journals.openedition.org/archipel/1156

DOI: 10.4000/archipel.1156

ISSN: 2104-3655

\section{Publisher}

Association Archipe

\section{Printed version}

Date of publication: 11 June 2019

Number of pages: $312-314$

ISBN: 978-2-910513-81-8

ISSN: 0044-8613

\section{Electronic reference}

Henri Chambert-Loir, "Urip Iku Urub : Untaian Persembahan 70 Tahun Profesor Peter Carey", Archipel [Online], 97 | 2019, Online since 16 June 2019, connection on 18 September 2021. URL: http:// journals.openedition.org/archipel/1156 ; DOl: https://doi.org/10.4000/archipel.1156 
siècle, il est sous-jacent dans nombre de chapitres et explicitement discuté à propos de Mbah Priok (181-212) et de Sunan Bayat (367-372). Ce dernier chapitre débouche sur une longue comparaison (372-409) entre les dévotions accomplies sur le site-même et dans la mosquée adjacente, avec la conclusion, solidement argumentée, que le pôle abangan-kramat (l'islam javanisant), majoritaire au lendemain de l'indépendance, est devenu aujourd'hui minoritaire, au profit du pôle santri-masjid (l'islam rigoureux ou tout bonnement orthodoxe). Parmi les nombreuses causes de ce renversement (urbanisation, éducation, internationalisation...), la tragédie de 1965 eut le rôle d'un catalyseur brutal. Avec la paradoxale constatation, cependant, que les pèlerinages sur les tombes n'ont cessé d'augmenter de façon spectaculaire depuis les années 1980 jusqu'à aujourd'hui, précisément par réaction envers la vague d'orthodoxie coercitive. Sur le plan religieux, les lieux saints (kramat) sont le refuge de la culture abangan (permissive), mais les tombes, plus ou moins habillées de signes d'orthodoxie ou de « santrification » (402), sont aussi visitées par des orthodoxes.

Au terme de ce cheminement ethnographique et savant à la fois, le lecteur découvre une forme de dévotion et de foi musulmane à l'opposé de tout ce que lui enseigne l'actualité internationale dont rendent compte les médias et même très éloignée de l'islam dont parlent les publications académiques sur l'Indonésie. La vénération des tombes, ainsi que d'autres sites sacrés, est indubitablement musulmane, mais elle s'inscrit dans un univers spirituel, qui représente un aspect essentiel du rapport des Javanais à l'au-delà. En filigrane à la visite des tombes, cet ouvrage présente une exploration de la relation historique, géographique et spirituelle, à Java, entre les mondes visible et invisible et entre le microcosme et le macrocosme.

Henri Chambert-Loir

Urip Iku Urub: Untaian Persembahan 70 Tahun Profesor Peter Carey ("La Vie comme un flambeau : Un collier d'offrandes pour les 70 ans du Professeur Peter Carey »), Jakarta : Kompas, 2019, xc-568 p., 120 illustrations, index. ISBN: 978-602412-534-9.

Comme souvent dans les hommages offerts à des professeurs d'université, un corps d'articles, dans ce volume, est constitué de souvenirs et de documents personnels, et un autre d'essais en rapport avec la discipline du professeur en question.

La première partie (c.130 p.) est consacrée à Peter Carey, à commencer par un texte autobiographique de celui-ci (illustré par 55 photographies éparses dans la première partie de l'ouvrage), qui fait une large place à ses origines et à son enfance.

Né en 1948 à Rangoon, où il passe son enfance, Peter Carey fait ses études en Angleterre, puis des études supérieures à Cornell et à Leyde; il fait durant deux ans des recherches à Java et devient professeur d'histoire de l'Europe moderne à Oxford, où il enseignera pendant 30 ans. Il passe sa thèse sur Diponegoro en 1977, mais elle ne sera publiée que trente ans plus tard parce que son parcours suit des détours imprévus. Il consacre, en effet, une grande partie de son temps, dans les années 19801990, à une campagne de soutien au Timor oriental, lors de la difficile transition qui 
conduit à l'indépendance et qui a provoqué chez lui une « crise morale » (p. 44). Il participe aussi à la fondation du Cambodia Trust en 1989 (qui fournit des prothèses aux victimes de mines personnelles), à celle d'une école bouddhiste en Angleterre en 1993, à celle d'un centre de méditation bouddhiste en Irlande en 2000, et à celle d'une École supérieure de santé à Jakarta vers 2009.

Enfin, il prend sa retraite d'Oxford en 2008 et s'installe à Jakarta. Il fait d'abord, durant quatre ans, l'expérience décevante d'un emploi bénévole au ministère («kafkaien », p. 46) de la Santé pour développer en Indonésie la compétence qu'il a acquise dans le domaine des prothèses de bras et jambes, mais, pour finir, il jouit d'une période de récolte de tous ses investissements de chercheur grâce au patronage - pour le moins inattendu - de la fondation de Hashim Djojohadikusumo (le frère et financier de Prabowo Subianto), qui lui permet de publier la traduction de sa thèse et de devenir professeur à l'Université Indonesia. La décision de vivre à Jakarta est beaucoup plus qu'un séjour supplémentaire à l'étranger. Peter Carey ambitionne de devenir citoyen indonésien et divers indices laissent penser qu'il s'idendifie comme javanais (ex. p. xxxiv, 110).

En marge de cette carrière professionnelle, quelques éléments de la vie privée font surface, dont une expérience de sortie du corps à l'âge de six ans, une autre de mort imminente à 22 ans, une rencontre quasiment mystique avec Diponegoro et plusieurs épisodes tragiques. De cette vie très remplie et faite de chapitres apparemment dissemblables, le texte autobiographique de Peter Carey explique la logique intellectuelle et spirituelle, avec la ferme conviction qu' " il n'y a pas de hasard dans l'existence » (p. 31).

Peter Carey est l'un des historiens de l'Indonésie les plus réputés et les plus brillants. Son travail sur Diponegoro, d'une exhaustivité exceptionnelle, est le résultat d'une recherche académique mais aussi mystique, les longues veilles dans les archives trouvant leur parallèle dans les pèlerinages et les méditations sur les sites où s'est accomplie la geste de Diponegoro. Peter Carey a acquis une aura particulière en Indonésie aujourd'hui, où il semble, pour certains, avoir avec le héros javanais un lien beaucoup plus fort que le seul rapport académique.

Cette partie contient le texte de la leçon inaugurale prononcée par Peter Carey à l'Université Indonesia, en décembre 2014, « en l'honneur de la Fondation Arsari Djojohadikusumo », sur le rôle de l'histoire dans la construction de l'avenir de l'Indonésie. Une bibliographie des travaux de Peter Carey, en fin de volume, complète cette section personnelle. Outre les travaux bien connus sur l'histoire de Java, se trouvent d'autres livres et articles, dont deux livres sur Timor et un sur la Birmanie.

Le travail de Peter Carey sur la Guerre de Java montre la dimension sociale et religieuse de la révolte de Diponegoro : la Guerre n'a pas été provoquée par la déception d'un prince dans ses ambitions personnelles; elle a résulté des dysfonctionnements de la société coloniale et elle visait à chasser l'envahisseur au nom du pouvoir de l'islam javanais. Son ouvrage majeur sur Diponegoro et la Guerre (The Power of Prophecy: Prince Dipanagara and the end of an old order in Java, 1785-1855) est paru à Leyde en 2007, puis en traduction indonésienne (Kuasa Ramalan: Pangeran Diponegoro dan Akhir Tatanan Lama di Jawa, 1785-1855) à Jakarta en 2012. Une version abrégée est parue en anglais (Destiny: The life of Prince Diponegoro, 1785-1855) et en indonésien (Takdir: Riwayat Pangeran Diponegoro, 1785-1855) en 2014. 
La parution de Kuasa Ramalan a donné lieu à de multiples célébrations : lancement de l'ouvrage, conférences, créations artistiques (un monologue théâtral, un jeu de figurines d'un wayang kulit Diponegaran, un roman, une bande dessinée, un tableau, un drame musical...), célébrations de divers anniversaires, une exposition, restauration du fameux tableau de Raden Saleh, rencontres des descendants de Diponegoro, élection du manuscrit de la Babad Diponegoro à l'inventaire de la Mémoire du monde, pèlerinages sur les sites de la geste de Diponegoro (à Java, Madura et Makassar), remise du bâton de pèlerinage de Diponegoro au ministère indonésien de la Culture (voir l'article de Pauline Lunsingh Scheurleer dans le présent volume), réalisation d'un documentaire sur Peter Carey. Tout ceci ressemble à un culte de Diponegoro, dont Peter Carey est le grand prêtre.

Dans la deuxième partie, d'une cinquantaine de pages, qui traite de la Guerre dans les arts, Werner Kraus, l'un des trois étrangers parmi les 24 auteurs qui ont participé à ce volume, montre d'ailleurs, avec force exemples, que Diponegoro fut célébré dès 1913, à Java, comme le héros de la lutte anti-coloniale, et donc de la liberté, ainsi que le champion de la cause du peuple, aussi bien par les nationalistes (Insulinde, Taman Siswa, PNI) que les communistes (ISDV, PKI) et les musulmans (Muhammadiyah, Permi). La quatrième section également traite de la Guerre.

Ces trois parties consacrées à Peter Carey, Diponegoro et la Guerre (13 essais) s'accompagnent de deux autres contenant 11 essais sur l'histoire de Java : l'ordre ancien $\left(17^{\mathrm{e}}-19^{\mathrm{e}} \mathrm{s}\right.$. ) et le nouveau $\left(19^{\mathrm{e}}-20^{\mathrm{e}} \mathrm{s}\right.$. $)$, soit avant et après la Guerre. La première de ces deux parties (Bagian III, «L'Ordre javanais ancien », 50 p.) est essentiellement consacrée à la confrontation entre Javanais et Européens : spéculations sur l'identité du personnage légendaire Juru Taman, alias Baron Sakeber ou Sakender dans les chroniques javanaises (Sri Margana), stratégie politique de Mataram face au pouvoir du clan Kajoran-Tempayat (Kuncoro Hadi), rencontre entre le Gouverneurgénéral néerlandais et le Sultan de Banten, en 1708, d'après un rapport néerlandais contemporain officiel (Hendrik E. Niemeijer), massacre des Chinois de Batavia de 1740 (Lilie Suratminto), et enfin domination de Raffles sur Yogyakarta grâce à une coopération avec les élites locales et le réseau chinois (Daya Negri Wijaya).

Quant à la deuxième de ces deux parties (Bagian V, « Après la chute de l'ordre javanais ancien », 110 p.), elle traite de la perception javanaise de la femme chinoise aux $19^{\mathrm{e}}$ et $20^{\mathrm{e}} \mathrm{S}$. (Didi Kwartanada), du destin, très romanesque, d'une princesse du Pakualaman dans la deuxième moitié du $19^{\mathrm{e}} \mathrm{s}$. (Sri Ratna Saktimulya), du règne de Mangkunegoro IV (Daradjadi), de la représentation de Surapati dans le roman de 1887 de Melati van Java (Sudibyo), de la dégradation du statut de dukun avec la diffusion de la médecine européenne (Martina Safitry) et de l'action éducative des sœurs franciscaines, à Mendut, au début du $20^{\mathrm{e}} \mathrm{s}$. (Eka Ningtyas).

Comme c'est souvent le cas dans ce genre de volume, les contributions sont de nature et de qualité diverses. Elles font preuve, cependant, d'une grande cohérence thématique. Les auteurs donnent la primauté aux sources locales, essentiellement javanaises, tout en manifestant la volonté d'intégrer les travaux et les avancées théoriques accomplis à l'étranger.

Peu de professeurs étrangers ont eu l'honneur d'un " festschrift " indonésien (Bernet Kempers, Zoetmulder, Lombard). Ce volume offre un portrait multiforme et haut en couleurs d'un professeur anglais, en même temps qu'un panorama des études indonésiennes sur l'histoire de Java du $17^{\mathrm{e}}$ au $20^{\mathrm{e}}$ siècles. 